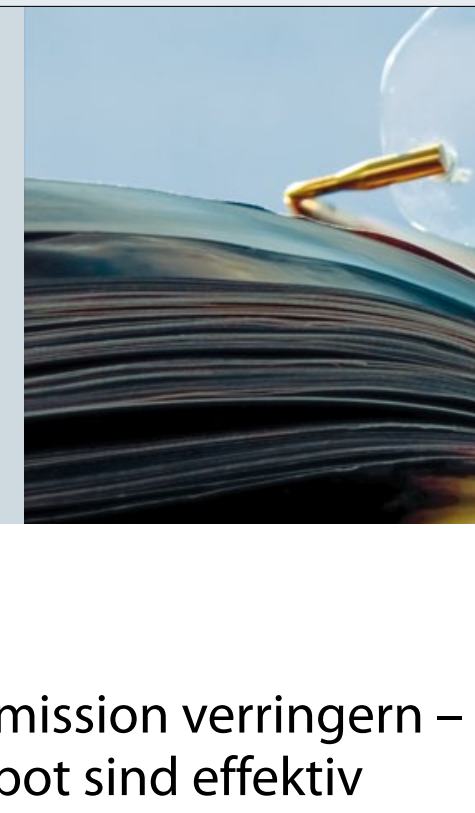
Antipsychotika als Depot sind effektiv

Patienten, die nach Remission Antipsychotika als Langzeitbehandlung erhalten, werden weniger oft „rückfällig" und verhalten sich weniger aggressiv.

D ie Metaanalyse von Leucht et al. [1] fasst relevante Arbeiten zum Thema „relapse-prevention“, also der Verhinderung erneuter psychotischer Episoden, innerhalb eines Jahres nach Remission zusammen. Berücksichtigt wurden 65 Studien mit insgesamt 6.493 Patienten, in denen immer ein Antipsychotikum mit Placebo verglichen wurde. Antipsychotika reduzierten signifikant das Risiko eines Rückfalls (27\% zeigten in einem Jahr unter Antipsychotika einen Rückfall, $64 \%$ unter Placebo, die Risk Ratio [RR, $27 \%: 64 \%]$ betrug 0,40 , number needed to treat benefit [NNTB] 3). Behandelte
Patienten mussten auch seltener stationär aufgenommen werden ( $10 \%$ vs. $26 \%$, RR: 0,38 , NNTB 5). Auch aggressive Handlungen kamen in der Verumgruppe seltener vor ( $2 \%$ vs. $12 \%$, RR: 0,27 , NNTB 11). Auf der anderen Seite zeigten Patienten unter Verum häufiger eine $\mathrm{Ge}$ wichtszunahme ( $10 \%$ vs. $6 \%$, RR: 2,07 ), Bewegungsstörungen (16\% vs. 9\%, RR: $1,55)$ und Sedierung ( $13 \%$ vs. $9 \%$, RR: 1,50). In Subgruppenanalysen zeigte sich, dass Depot-Antipsychotika effektiver als orale Antipsychotika waren. Zwischen Erst- und Zweitgenerationsantipsychotika ergaben sich keine Unterschiede.
Kommentar: Diese Arbeit zeigt eindrucksvoll die Überlegenheit von Antipsychotika versus Placebo in der Langzeitbehandlung der Schizophrenie. Jeder der untersuchten Effektivitätsparameter (mit Ausnahme sozialer Integration, Lebensqualität) zeigten diese Überlegenheit.

In einem begleitenden Editorial [2] wurde dieser Beitrag gewürdigt und angesichts der auch festgestellten Nebenwirkungen noch weiterführende Kosten-Nutzen-Analysen angemahnt.

Prof. Dr. med. Hans-Peter Volz

1. Leucht $S$ et al. Antipsychotic drugs versus placebo for relapse prevention in schizophrenia: a systematic review and meta-analysis. Lancet 2012; 379: 2063-71

2. Von Os J, Howes OD. Antipsychotic drugs for prevention of relapse. Lancet 2012; 379: 2030-31

\title{
Raus aufs Land? - Je städtischer die Umgebung, desto höher das Schizophrenierisiko
}

Sollte man Menschen, die zur Hochrisikogruppe gehören, präventiv mehr Ausflüge aufs Land oder sogar einen Umzug verordnen?

$\mathrm{D}$ ie Assoziation zwischen Urbanität und Schizophrenierisiko ist ein gut etablierter Befund. Dieses Risiko wird in den einzelnen Studien mit der Incidence Risk-Ratio (IRR) angegeben. Meist wird die IRR als Resultat des Vergleichs der ländlichsten Region mit der städtischsten Region angegeben.

Die Autoren verwendeten nun in ihrer Metaanalyse die Daten aus vier Untersuchungen, die Urbanität als Risikofaktor für das Entstehen einer Schizophre- nie sehen, und unterzogen diese mathematischen Transformationen (Regressionsanalyse, logistische Regression). Danach zeigte sich ein linearer Zusammenhang zwischen dem Logarithmus der IRR und dem Grad der Urbanität. Das Risiko an Schizophrenie zu erkranken, ist bei Gesamtschau dieser Daten in den städtischsten Regionen um 2,37fach höher als in den ländlichsten Regionen. Diese Ergebnisse waren nicht abhängig von der diagnostischen Ein- ordnung („,enge" Schizophreniediagnose, ,breite“ Psychosediagnose), von der Methode der Quantifizierung von Urbanität (Einwohnerzahl, Populationsdichte) und von der Zeit der Exposition (Geburt und Aufwachsen oder Ausbruch der Krankheit).

Kommentar: Erstaunlich an dieser Arbeit ist, dass der Zusammenhang zwischen Urbanität und dem Risiko der Schizophrenie einem stetigen Zusammenhang folgt, was an eine Dosis-Wirkungsbeziehung erinnert und die Robustheit dieses Befundes zeigt.

Prof. Dr. med. Hans-Peter Volz

Vassos E et al. Meta-analysis of the association of urbanicitiy with schizophrenia.

Schizophr Bull 2012; 38: 1118-23 